\title{
Electron Beam Induced Radiation Damage in Nafion and the Lifetime of Fuel Cells
}

\author{
Qianping He*, David C. Joy**,***, David J. Keffer* \\ * Dept. of Chemical and Biomolecular Engineering, University of Tennessee, Knoxville, TN 37996 \\ ** Dept. of Materials Science and Engineering, University of Tennessee, Knoxville, TN 37996 \\ *** Center for Nanophase Materials Science, Oak Ridge National Laboratory, Oak Ridge, TN 37831
}

Polymer electrolyte membrane fuel cells (PEMFCs) are a potentially viable, clean, and renewable source of energy. There are numerous technical challenges that must be overcome in order to achieve significant market penetration. The interface between the electrode and electrolyte remains one of the areas of greatest ignorance. A molecular-level understanding of fuel cell structure will be very helpful to understand the electron/proton transportation mechanism at the electrode/electrolyte interface, see Fig. 1. SEM and TEM are two powerful tools commonly used to gain molecular level information about the electrode/electrolyte interface structure. However, Schneider [1], has reported that Nafion (used as electrolyte in the PEMFC) is susceptible to radiolysis during SEM imaging (Fig. 2.), and suggested that such damage was dependent on the microstructure of the substance being viewed. Because beam induced damage could have major implications for transport phenomena occurring at the electrolyte/electrode interface of PEMFC and could possibly suggest one of the possible mechanisms of fuel cell aging, we have investigated the response of Nafion to electron beam irradiation.

In the work described here, high angle annular dark field (HAADF) STEM images of our interface samples were recorded as a function of the integrated beam dose using a Zeiss MERLIN SEM operated in scanning transmission (STEM) mode. The magnitude of the HAADF signal is linearly proportional to the mass thickness of the specimen [2]. So thicker regions of the sample or areas with higher density will appear bright, whilst a hole through the sample in the beam path will appear darker. To make measurements the probe beam, containing a known beam current, is scanned at TV rates in a square raster a few micrometers in size across the Nafion sample for time periods varying from a few seconds up to of the order of one minute. An image of the exposed area is then recorded and, during the photo-recording, the beam is blanked for one or two seconds to provide a zero-signal ("black level") reference. The recorded STEM image is then analyzed by a histogram which indentifies both the zero signal baseline value and the signal level in the irradiated region (Fig. 3.), which permits the brightness of the irradiated area to properly determined. This procedure is then repeated, as required, to increase the deposited beam dose while simultaneously measuring the change in STEM image brightness. The relative change in sample thickness with irradiation can then be found by plotting the signal intensity as a function of the beam dose deposited. An absolute measurement of the damage rate versus dose can be produced by using a mass standard, such as ferritin, to calibrate the sensitivity of the HAADF signal under the given experimental conditions.

\section{References}

[1] Roxanne Schneider, Scanning Electron Microscopy Studies of Nafion Deformation into Silicon Micro-Trenches for Fuel Cell Applications, Ph.D. Thesis, Princeton University, New Jersey, 2008. 
[2] Joy D C et al., Scanning Microscopy. 10 (1996) 653.

[3] This work was supported by the STAIR program at the University of Tennessee, funded by NSF under agreement number: DGE 0801470.

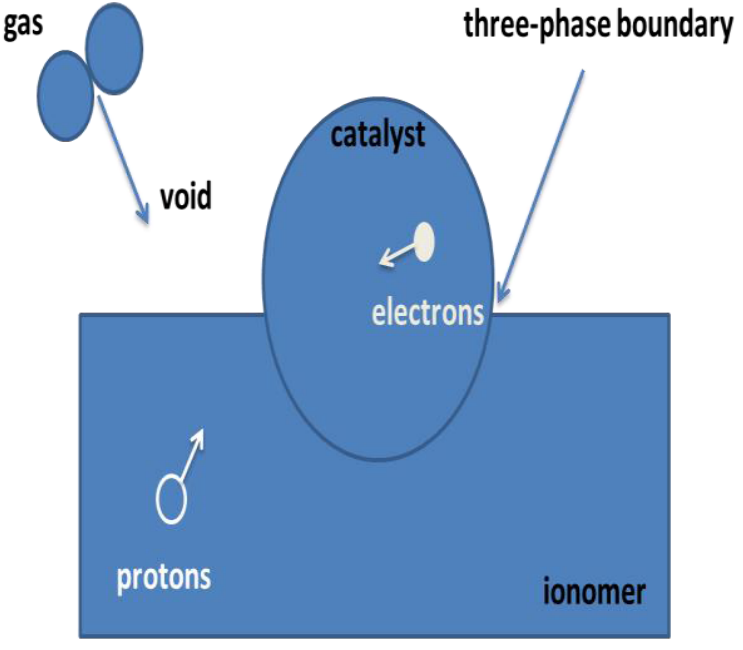

FIG. 1. An illustration of the three phase boundary at which the electrochemical reaction takes place in a PEMFC.

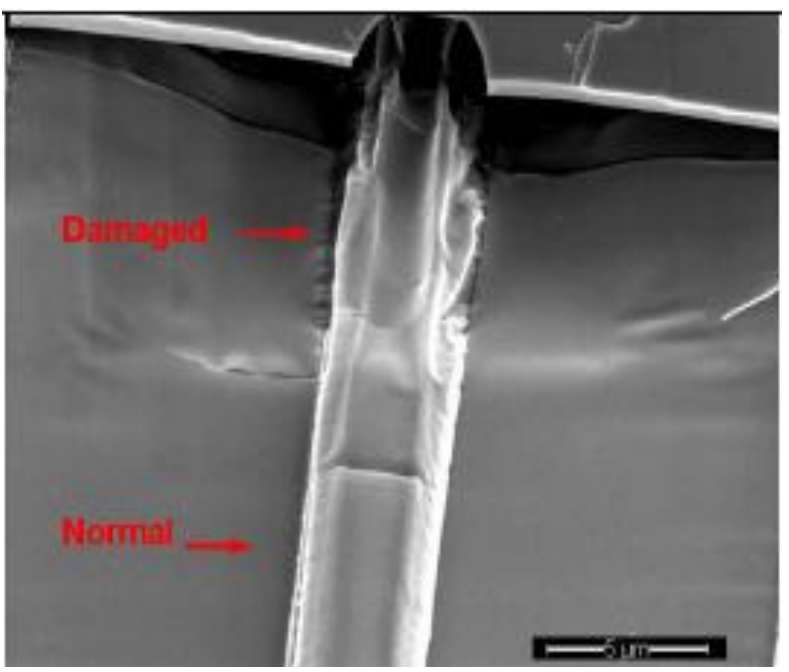

FIG. 2. A 5000X image of both the damaged and undamaged portion of a feature in the Nafion sample dry pressed at $140{ }^{\circ} \mathrm{C}[1]$.

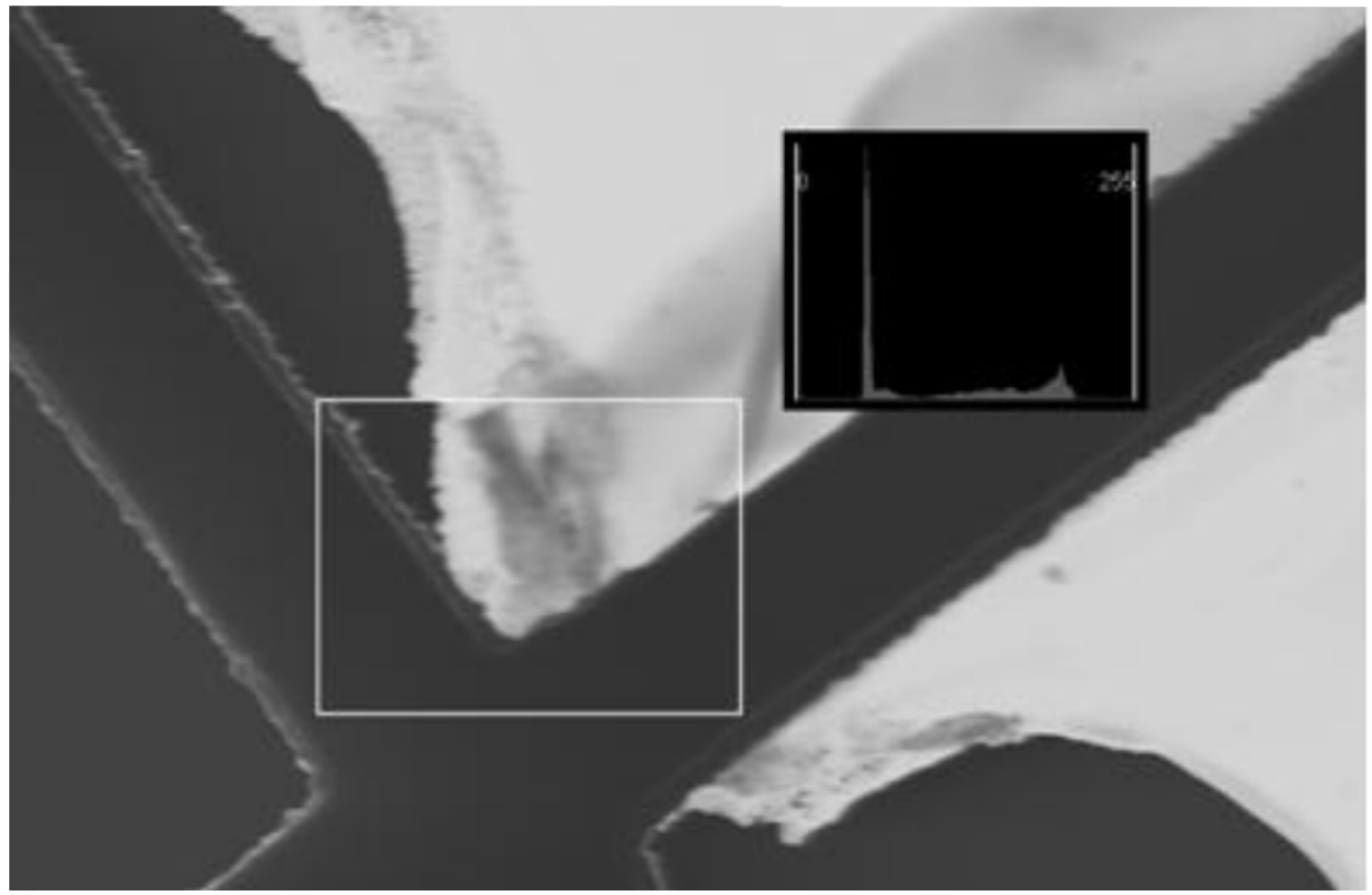

FIG. 3. 30kev HAADF STEM image of a Nafion sample showing the histogram obtained from the highlighted, rectangular, irradiated area. The image field of view is $70 \mu \mathrm{m}$ in width. 